OPEN ACCESS

Edited by:

Lisa A. Morici,

Tulane University School of Medicine,

United States

Reviewed by:

Michael J. Schurr

University of Colorado Denver,

United States

Michelle B. Visser,

University at Buffalo, United States

*Correspondence:

Eric Déziel

eric.dezie/@iaf.inrs.ca

Specialty section:

This article was submitted to Molecular Bacterial Pathogenesis, a section of the journal

Frontiers in Cellular and Infection

Microbiology

Received: 16 November 2018

Accepted: 01 February 2019

Published: 28 February 2019

Citation:

Coulon PML, Groleau M-C and

Déziel E (2019) Potential of the

Burkholderia cepacia Complex to

Produce

4-Hydroxy-3-Methyl-2-Alkyquinolines.

Front. Cell. Infect. Microbiol. 9:33.

doi: 10.3389/fcimb.2019.00033

\section{Potential of the Burkholderia cepacia Complex to Produce 4-Hydroxy-3-Methyl-2-Alkyquinolines}

\author{
Pauline M. L. Coulon, Marie-Christine Groleau and Eric Déziel* \\ Institut Armand Frappier, Institut National de la Recherche Scientifique, Laval, QC, Canada
}

A few Burkholderia species, especially Burkholderia pseudomallei, Burkholderia thailandensis, Burkholderia ambifaria, and Burkholderia cepacia, are known to produce and release various 4-hydroxy-3-methyl-2-alkylquinolines (HMAQs), a family of molecules analogous to the 4-hydroxy-2-alkylquinolines [aka 2-n-alkyl-4(1H)-quinolones] of Pseudomonas aeruginosa, which include the Pseudomonas quinolone signal (PQS). However, while these exoproducts play several roles in $P$. aeruginosa virulence and survival, the available literature is very limited on their distribution and function in Burkholderia. In this perspective article, we studied the distribution of the hmqABCDEFG operon, which encodes the enzymes involved in the biosynthesis of HMAQs, in the Burkholderia cepacia complex (Bcc) group. Based on the available sequence data, about one third of Bcc species carry a homolog of the hmaABCDEFG, and not all sequenced strains in a given species possess this operon. Looking at the synteny of genes surrounding the $h m 9 A B C D E F G$ operon, we found that for some species, the operon seems to have been deleted or replaced by other genes. Finally, we review the literature on the possible function of HMAQs. Understanding the Hmq system may provide clues concerning their functions in Bcc.

Keywords: $h m q A B C D E F G$ operon ( $h m q)$, quorum sensing, synteny, 4-hydroxy-2-alkylquinolines (HMAQ), alkylquinolones, Pseudomonas aeruginosa, pqsABCDE operon

\section{INTRODUCTION}

In 1992 several species originally classified as Pseudomonas defined the new Burkholderia genus (Yabuuchi et al., 1992). This genus now comprises more than $60 \mathrm{Gram}$-negative bacterial species, which fit into two clades: the plant-associated beneficial and environmental one or the pathogenic one (Surez-Moreno et al., 2012; Eberl and Vandamme, 2016). The plant-associated beneficial and environmental clade has recently been renamed Paraburkholderia because these bacteria lack biomolecular markers specific to pathogenic strains belonging to Burkholderia genus (Sawana et al., 2014). However, this split is controversial since some strains can be both pathogenic to animals and beneficial to plants (Eberl and Vandamme, 2016). The Burkholderia pathogenic clade is composed of plant, animal and human pathogens separated in two well-known groups: the "pseudomallei" group (B. pseudomallei, B. mallei and the environmental strain and study model B. thailandensis) and the opportunist pathogen species forming the Burkholderia cepacia complex (Bcc) (Surez-Moreno et al., 2012).

Bacteria belonging to the Bcc are mostly found in the rhizosphere, soil and water (Eberl and Vandamme, 2016; Loveridge et al., 2017). Bcc species are of interest in the industrial 
and agricultural fields, for instance for their potential in bioremediation (e.g., Burkholderia vietnamiensis), in plant growth promotion (e.g., Burkholderia ambifaria) and also for their capacity to produce an array of secondary metabolites [reviewed by (Vial et al., 2007)]. However, the realization that many Bcc species are responsible for serious chronic infections among immunosuppressed patients in general, notably those suffering from cystic fibrosis (CF) or chronic granulomatous disease (CGD), has put a hold on their biotechnological use, especially in agriculture (Vial et al., 2011). Indeed, because such infections are highly transmissible between patients and typically highly antibiotic resistant, they are often fatal (Gold et al., 1983; Gilligan, 1991; Govan et al., 1993; Speert et al., 1994; Govan and Deretic, 1996; LiPuma, 1998).

Bcc species produce various virulence determinants such as exopolysaccharides, siderophores and antimicrobials and can adopt several social behaviors such as swarming motility and biofilm formation. These are controlled by quorum-sensing (QS) (Kang et al., 1998; Lewenza et al., 1999; Richau et al., 2000; Huber et al., 2001; El-Banna and Winkelmann, 2002; Aguilar et al., 2003). QS is a cell-to-cell communication system used by bacterial populations to sense their density and thus control the transcription of certain genes in a coordinated manner (Fuqua and Greenberg, 1998). In fact, this signaling system allows bacteria to optimize colonization, to interact with their hosts and to better resist to stresses (Stewart and Costerton, 2001; Juhas et al., 2005).

In the $\mathrm{Bcc}$, CepR is the main QS transcriptional regulator, which is activated by the autoinducing signal N-octanoylhomoserine lactone $\left(\mathrm{C}_{8}\right.$-HSL), the product of CepI (McKenney et al., 1995; Lewenza et al., 1999; Lewenza and Sokol, 2001). Depending on the species, Bcc have at least two cep (luxlike) systems (Choudhary et al., 2013), similar to the las and rhl systems in Pseudomonas aeruginosa. Interestingly, Pesci et al. (1999) reported a third signal they called the Pseudomonas quinolone signal (PQS) also produced in the latter bacterial species. It was then found that PQS actually belongs to a large family of extracellular molecules called 4hydroxy-2-alkylquinolines (HAQ), also referred to as 2-n-alkyl4(1H)-quinolones (Déziel et al., 2004). Gallagher et al. (2002) identified the $p q s A B C D E$ polycistronic operon as required for the production of these HAQs in $P$. aeruginosa. The pqs system was confirmed as a bona fide QS system when PQS, and its biosynthetic precursor 4-hydroxy-2-heptylquinoline (HHQ), were shown to act as autoinducing ligands of the transcriptional regulator MvfR (also known as PqsR) (Wade et al., 2005; Xiao et al., 2006). MvfR controls the expression of pqsABCDE (Déziel et al., 2004) resulting in an autoinducing loop. Unexpectedly, a few Burkholderia strains were then reported to produce minute levels of HHQ (but not PQS) and to carry a cluster of pqsABCDE homologs (Diggle et al., 2006). Subsequently, it was found that the main products in these Burkholderia strains are actually 4-hydroxy-3-methyl-2-alkylquinolines (HMAQs), principally distinct from $P$. aeruginosa HAQs by the presence of a methyl at the $3^{\prime}$ position and the predominance of an unsaturated alkyl side chain, among congeners (Vial et al., 2008), explaining why Diggle et al. (2006) had only detected very low concentrations of HHQ (Figure 1A). This biosynthetic operon was accordingly named $h m q A B C D E F G$, with the additional encoded $\mathrm{HmqF}$ and $\mathrm{HmqG}$, respectively, responsible for the unsaturation (Agarwal et al., 2012) and the methylation (Vial et al., 2008) of HMAQs. It should be mentioned that no congener corresponding to PQS, thus with a hydroxy substitution at the 3 position, has been detected in any Burkholderia culture, in agreement with the absence of a $p q s H$ homolog. As with the $p q s$ system in $P$. aeruginosa, interactions between the $h m q$ and cep systems have been reported in B. ambifaria (Vial et al., 2008; Chapalain et al., 2017). While the production of some HMAQ congeners by a few Burkholderia isolates has been reported (Diggle et al., 2006; Mori et al., 2007; Vial et al., 2008; Kilani-Feki et al., 2011, 2012; Mahenthiralingam et al., 2011; Li et al., 2018), no studies have yet systematically investigated the prevalence of the $h m q$ system and the capacity to produce HMAQs in the genus Burkholderia. In this perspective article, we review the knowledge on the $h m q$ system, update the HMAQ biosynthesis pathway and investigate the distribution of the $h m q A B C D E F G$ operon in Burkholderia and especially in Bcc.

\section{DISTRIBUTION OF THE $h m q A B C D E F G$ OPERON IN THE BURKHOLDERIA GENUS}

Based on complete and draft genome sequences available in the Burkholderia Genome database (http://www.burkholderia. com), we have performed two complementary analyses with hmqABCDEFG of B. ambifaria strain AMMD as a reference: (1) an alignment sequence analysis () and (2) an orthology analysis. The BLAST analysis was run for the nucleic acid sequence of each gene composing the $h m q$ operon, using the default settings (Johnson et al., 2008; Winsor et al., 2008). The orthology analysis consisted in examining the similarity of the protein sequence and the flanking gene regions by the pair-wise Diamond searches (Winsor et al., 2008; Buchfink et al., 2015). While that method may be more accurate than a BLAST analysis, an orthology analysis could miss some positive results if the hmqABCDEFG operon is in a different genomic location than the reference. Thus, a combination of both methods was selected.

Using the two analyses, we screened: (1) the seven genes composing the hmqABCDEFG operon based on the percentage of identity and (2) the species having all seven genes present in their genome. Sequences of the $h m q A B C D E F G$ operon are well conserved, with a nucleotide identity between 70 and $100 \%$. Importantly, we only found instances of a $h m q G$ homolog in a genome if it belonged to a $h m q A B C D E F G$ operon.

Based on both analysis methods, the hmqABCDEFG operon was found in 6 out of 21 Bcc species: B. cepacia, B. ambifaria, B. pyrrocinia, B. ubonensis, B. contaminans, and B. stagnalis, plus in some still unclassified Burkholderia spp.--having at least one sequenced genome present in the Burkholderia.com database (Table 1). Furthermore, some strains of B. lata, and B. territorii appear to have the $h m q A B C D E F G$ operon too, but this was only found via the BLAST analyses. Actually, the percentage of 


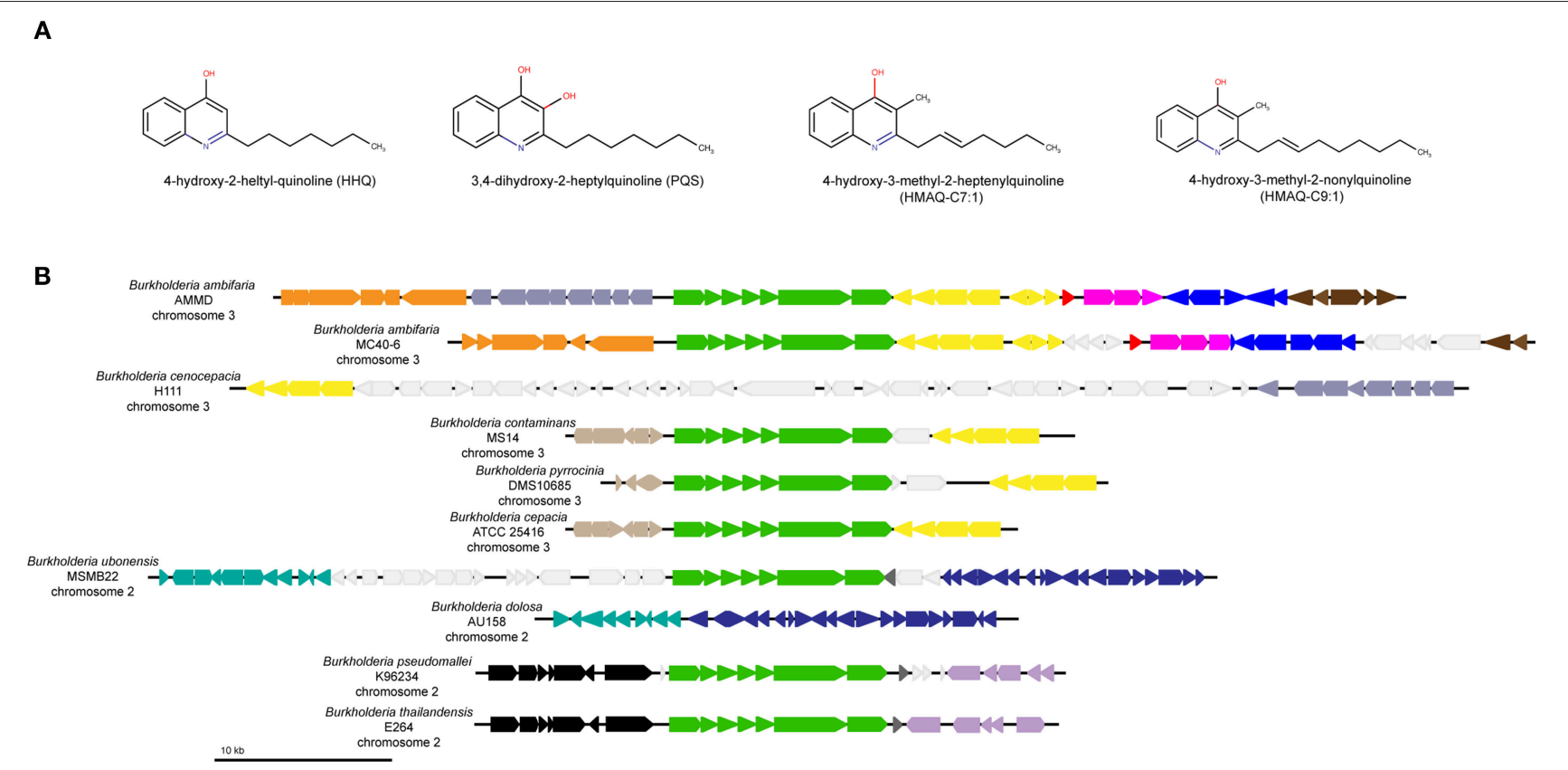

FIGURE 1 | HAQ molecules and the synteny of genes surrounding the hmqABCDEFG operon in Burkholderia cepacia complex species. (A) Representation of HAQ molecules found in $P$. aeruginosa or/and in Burkholderia. (B) Synteny of $h m q A B C D E F G$ operon in Bcc: each color represents a different synteny group determined by MAUVE software. Only the closest genes of the hmaABCDEFG operon have been shown. The hmqABCDEFG operon is represented in green color. Light gray color represents variable metabolic genes without synteny.

strains having the putative operon is higher by BLAST analysis than by orthology analysis. The hmqABCDEFG operon shows strong nucleic acid identity between the strains and within the genus Burkholderia. In fact, the average nucleic identities for each gene are between 78.5 and $83.9 \%$ compared to the B. ambifaria AMMD operon sequences (Supplementary Table 1). All these data show that (1) about one third of Bcc species carry the hmqABCDEFG operon and (2) within a species not all of the strains carry it.

A Bayesian phylogeny analysis, based on the hmqABCDEFG genes, shows extensive concordance with strain speciation, implying that this operon has been inherited from the common ancestor of Burkholderia rather than the results of recent gene transfer (Supplementary Figure 1). The presence of homologous operons in the evolutionary distant genera Burkholderia and Pseudomonas suggests a possible past horizontal gene transfer event, typically characterized (1) by a different \%GC, (2) mobile elements insertion, and (3) genomic islands (Lawrence and Ochman, 2002; Juhas et al., 2009; Ravenhall et al., 2015). A similarly high \%GC between Burkholderia (65\%) and $P$. aeruginosa (67\%) prevents the use of $\% \mathrm{GC}$ to infer lateral gene transfer between these species. ISfinder (http://www-is.biotoul.fr) was used to find already known repeat sequences as indications of mobile elements insertion (Siguier et al., 2006). Islandviewer 4 (http://www. pathogenomics.sfu.ca/islandviewer) was used to predict genomic island on the third chromosome of $B$. ambifaria AMMD genome, and the second chromosome of $B$. thailandensis E264 and B. pseudomallei K96243 genomes (Bertelli et al.,
2017). None of these methods revealed clear indications of horizontal gene transfer of the hmqABCDEFG operon and its surrounding genes.

\section{THE SYNTENY OF THE hmqABCDEFG OPERON IN BURKHOLDERIA}

Given that the hmqABCDEFG operon has a heterogeneous distribution in $\mathrm{Bcc}$, we decided to examine its genomic context. The number of complete genome sequences being limited, the analyses was performed on $B$. ambifaria AMMD, $B$. ambifaria MC40-10, B. cepacia ATCC25416, B. contaminans MS14, B. pyrrocinia DSM 10685, B. ubonensis MSMB22, B. pseudomallei K96243, and B. thailandensis E264, all available in the Burkholderia Genome database (Winsor et al., 2008).

A multiple whole-genome alignment using progressiveMAUVE software was done to view conserved regions surrounding the $h m q A B C D E F G$ operon (Figure 1B) (Darling et al., 2010). By this analysis, we defined three groups: (1) B. ambifaria, (2) B. contaminans, B. pyrrocinia, B. cepacia, and (3) B. ubonensis, B. cenocepacia H111, and B. dolosa AU158 strains. Members of this third group lack the $h m q A B C D E F G$ operon in their genomes, are also included in the comparison to show a possible gene rearrangement. Synteny in genes surrounding the operon confirms the absence of the operon in B. cenocepacia $\mathrm{H} 111$ in which it is replaced by a number of metabolic genes. Also, a high similarity exists between the neighboring genes of $B$. dolosa 
TABLE 1 | The hmqABCDEFG operon distribution in Burkholderia cepacia complex.

\begin{tabular}{|c|c|c|c|c|}
\hline \multirow[t]{2}{*}{ Species } & & \multirow{2}{*}{$\begin{array}{l}\text { Bcc strains included in } \\
\text { Burkholderia Genome DB } \\
\text { [Complete genomes] }\end{array}$} & \multicolumn{2}{|c|}{$\begin{array}{c}\text { Analysis of the } h m q A B C D E F G \text { operon's } \\
\text { distribution by sequence }\end{array}$} \\
\hline & & & $\begin{array}{l}\text { Orthology analysis } \\
\text { (DIAMOND) }\end{array}$ & $\begin{array}{c}\text { Alignment analysis } \\
\text { (BLASTN) }\end{array}$ \\
\hline \multirow{22}{*}{$\begin{array}{l}\text { Burkholderia } \\
\text { cepacia complex } \\
\text { (Bcc) }\end{array}$} & Burkholderia cepacia (genomovar I) & $337[24]$ & 42 & 77 \\
\hline & Burkholderia multivorans (genomovar II) & $56[7]$ & - & - \\
\hline & Burkholderia cenocepacia (genomovar III) & $243[15]$ & - & - \\
\hline & Burkholderia stabilis (genomovar IV) & - & - & - \\
\hline & Burkholderia vietnamiensis (genomovar V) & $41[6]$ & - & - \\
\hline & Burkholderia dolosa (genomovar VI) & $2[1]$ & - & - \\
\hline & Burkholderia ambifaria (genomovar VII) & $6[2]$ & 2 & 3 \\
\hline & Burkholderia anthina (genomovar VIII) & $8[-]$ & - & - \\
\hline & Burkholderia pyrrocinia (genomovar IX) & $4[1]$ & 1 & 3 \\
\hline & Burkholderia ubonensis (genomovar X) & $292[6]$ & 75 & 283 \\
\hline & Burkholderia latens (BCC1) & $2[1]$ & - & - \\
\hline & Burkholderia diffusa (BCC2) & $12[1]$ & - & - \\
\hline & Burkholderia arboris (BCC3) & - & - & - \\
\hline & Burkholderia seminalis (BCC7) & $3[1]$ & - & - \\
\hline & Burkholderia metallica (BCC8) & $1[1]$ & - & - \\
\hline & Burkholderia lata (group K) & $4[2]$ & - & 2 \\
\hline & Burkholderia contaminans (group K, BCCAT) & $7[1]$ & 2 & 3 \\
\hline & Burkholderia pseudomultivorans & $9[1]$ & - & - \\
\hline & Burkholderia stagnalis (BCC B) & $64[1]$ & 32 & 63 \\
\hline & Burkholderia territorii (BCC L) & $33[1]$ & - & 2 \\
\hline & Burkholderia paludis & - & & \\
\hline & Burkholderia sp. & 59 [18] & 2 & 11 \\
\hline \multicolumn{2}{|l|}{ Total of Bcc strains } & $1257[91]$ & 166 & 447 \\
\hline \multicolumn{2}{|l|}{ B. pseudomallei } & $677[75]$ & 27 & 655 \\
\hline \multicolumn{2}{|l|}{ B. thailandensis } & $28[15]$ & 13 & 22 \\
\hline \multicolumn{2}{|c|}{ Total of pseudomallei group strains } & 705 [90] & 284 & 677 \\
\hline \multicolumn{2}{|l|}{ Total of strains } & 1962 [181] & 450 & 1123 \\
\hline
\end{tabular}

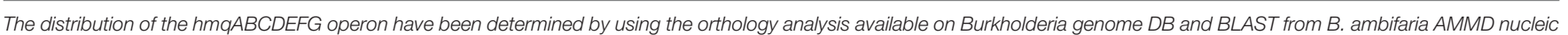

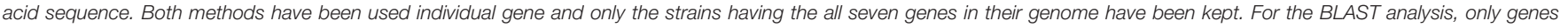
having a high identity with the reference have been kept. The sign "-" means that no sequences were available.

AU158 and B. ubonensis MSMB22. In fact, in B. dolosa AU158 the $h m q A B C D E F G$ seems to have been deleted from or not yet integrated into the genome of this strain. The lack of available complete sequences limits the synteny analysis, more comparison could help us to have a better understanding of the hmqABCDEFG distribution.

\section{THE HMQ SYNTHESIS PATHWAY IN BURKHOLDERIA}

In Burkholderia, the hmq system was found first on the second chromosome of four Burkholderia pseudomallei (K96243, $576,10276,844), B$. thailandensis E30 and on the third chromosome of $B$. cenocepacia J415, to be homologous to the Pseudomonas Quinoline Signal (PQS) system found in $P$. aeruginosa (Diggle et al., 2006). The hmq system produces 4-hydroxy-2-heptylquinoline (HHQ) but not 3,4dihydroxy-2-heptylquinoline (PQS) signaling molecules (Diggle et al., 2006). In fact, the strains produce the methylated 4hydroxy-3-methyl-2-alkylquinolines (HMAQs) instead of PQS molecules (Vial et al., 2008). The hmqABCDEFG operon encodes the $h m q$ system and has two more genes than the $p q s A B C D E$ operon in $P$. aeruginosa meaning that the HMAQ biosynthesis and PQS biosynthesis pathways are similar except for the $\mathrm{HmqF}$ and $\mathrm{HmqG}$ additional functions [Figure 2; (Vial et al., 2008)].

In fact, the putative anthraniloyl-CoA synthase encoded by $h m q A$, allows anthraniloyl-CoA synthesis from anthranillic acid, coming from tryptophan or chorismic acid by the KynABU catabolism pathway and the TrpEG biosynthesis pathway, respectively (Farrow and Pesci, 2017). The putative 3-oxoacyl-ACP-synthase (HmqD), binding the anthraniloyl-CoA to transfer a SCoA fatty acid or an acetate 
Toulon et al.

HMAQ Biosynthesis Genes in Rc

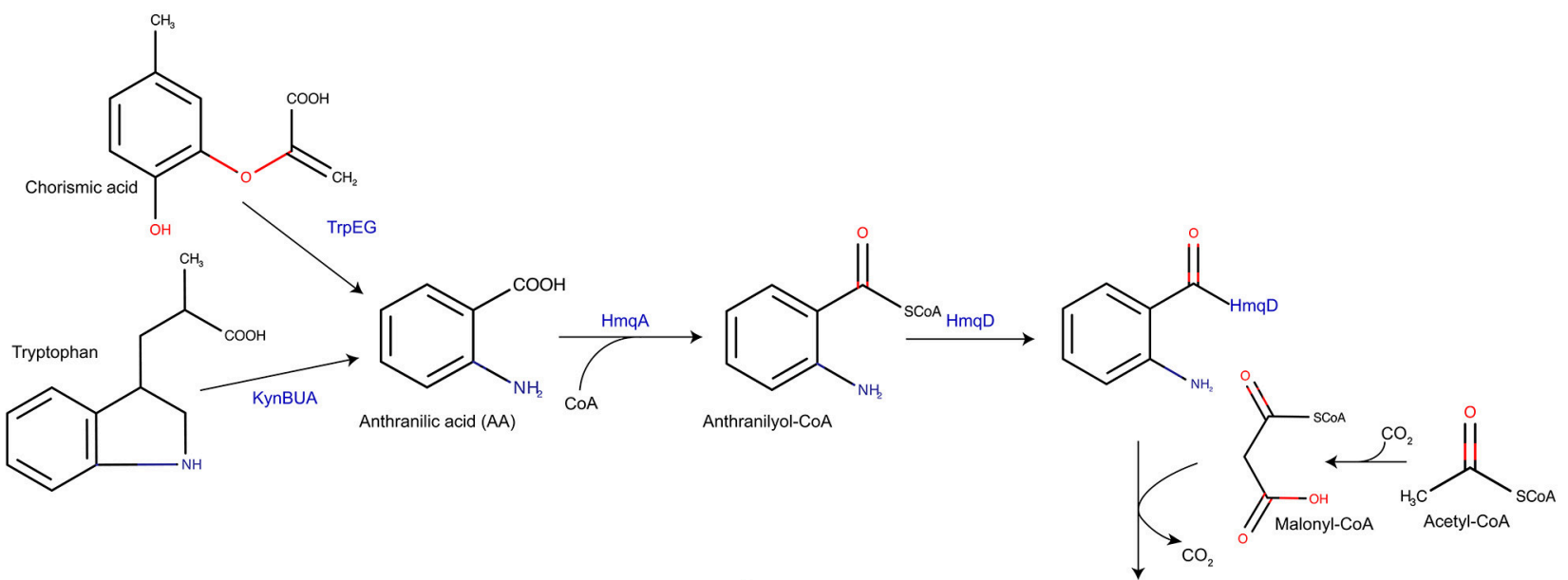<smiles>Oc1cc(O)c2ccccc2n1</smiles><smiles></smiles>

2,4-dihydroxyquinoline (DHQ)
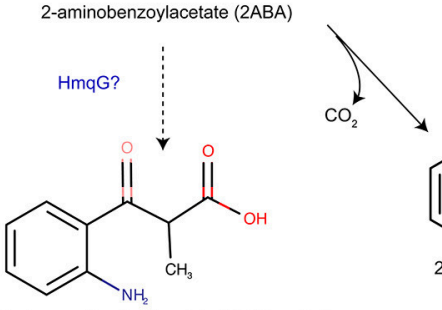<smiles>Cc1c(O)nc2ccccc2c1O</smiles>

2,4-dihydroxy-3-methylquinoline (DHMQ)

2-aminobenzoyl(methyl)acetate (2ABAmethyl)

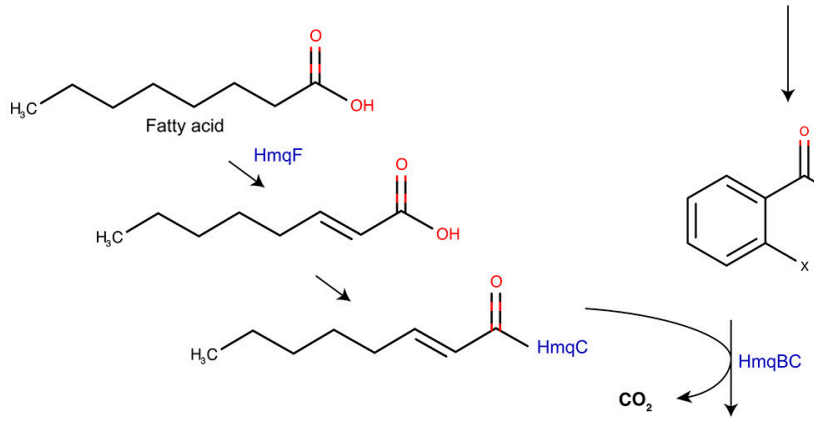<smiles>[X]c1ccccc1C(=O)C(C)C(=O)/C=C/CCCCC</smiles>

$\mathrm{X}=\mathrm{NH}_{2}, \mathrm{NHOH}(\mathrm{HmqL})$<smiles>CCCCC/C=C/c1nc2ccccc2c(O)c1C</smiles><smiles>CCCCC/C=C/c1c(C)c(O)c2ccccc2[n+]1[O-]</smiles>

4-hydroxy-3-methyl-2-heptylquinoline (HMAQ-C7:1) 4-hydroxy-2heptylquinoline $\mathrm{N}$-oxide (HMAQC7:1-N-Oxide)

FIGURE 2 | An updated version of the Hq biosynthesis pathway based on Vial et al. (2008). Proposed Hmo biosynthesis pathway, based on HAQ biosynthesis in P. aeruginosa, produces $\mathrm{HHQ}$ and $\mathrm{HMAQ}$ both with a saturated or unsaturated acyl chain. HmqF adds the unsaturation on the fatty acid. HmqG may ethylate 2-ABA to form 2-ABAmethyl which will form either HMAQ and HMAQ-N-oxides but also DHMQ in lower quantity. Note that only B. thailandensis is able to produce HQNO molecules.

Frontiers in Cellular and Infection Microbiology | www.frontiersin.org

5

February 2019 | Volume 9 | Article 33 
to form 2-aminobenzoylacetate-CoA (Zhang et al., 2008; Dulcey et al., 2013). In addition, playing an active role in QS, the putative metallo- $\beta$-lactamase $\mathrm{HmqE}$ should favor the HHQ pathway production, because of the phenotype restoration in a $p q s E$ mutant by adding $h m q E$ (Diggle et al., 2006; Farrow et al., 2008; Rampioni et al., 2010, 2016; Folch et al., 2013; Drees and Fetzner, 2015). However, a pqsE mutant is still able to produce HHQ/PQS showing that PqsE is not been essential for PQS biosynthesis. Moreover, the $\beta$-cetodecanoyl-ACP synthases $\mathrm{HmqB}$ and $\mathrm{HmqC}$ should form a complex like PqsBC [5DWZ; www.rcbs.org; Drees et al., 2016], considering the high similarity between the N-terminal amino acid sequence between $\mathrm{HmqB}$ and $\mathrm{PqsB}$. By protein sequence analysis performed with Chimera [UCSF Chimera; (Pettersen et al., 2004)], this conserved amino acid sequence should allow the interaction between $\mathrm{HmqB}$ and $\mathrm{HmqC}$ by forming the same structure $\mathrm{PqsB} / \mathrm{PqsC}$, presumably. The two supplementary genes $h m q F$ and $h m q G$ encode, respectively, putative AMP-dependant synthase ligase and methylase. $\mathrm{HmqF}$ is responsible for the unsaturation on the alkyl sidechain while $\mathrm{HmqG}$ is responsible for the methylation of the molecule (Vial et al., 2008; Agarwal et al., 2012).

Burkholderia species do not carry $\mathrm{PqsH}$ homologous in their genomes, which explains the absence of the synthesis of 3 hydroxyl-HHQ molecules, such as PQS (Déziel et al., 2004). However, HmqL, an homolog of PqsL, has been found in $B$. thailandensis E264. This protein is a putative flavin adenine dinucleotide mono-oxygenase allowing the production of $\mathrm{N}$ oxide HMAQs (Vial et al., 2008; Butt et al., 2016).

\section{HMAQS PRODUCTION}

The first HMAQ congener reported, named burkholone, was identified in culture broth of the environmental strain Burkholderia sp. QN15488 (Mori et al., 2007). Vial et al. (2008) described the production of three HMAQ families in B. pseudomallei 1026b, B. thailandensis E264, and B. ambifaria HSJ1, for a total of 19 different HMAQ congeners. This is without mentioning congeners without a methyl at the 3 position, thus similar to those produced by $P$. aeruginosa (Déziel et al., 2004). While it was originally believed that among Bcc species, only clinical B. ambifaria strains produce these molecules (Vial et al., 2008, 2009), it was later reported that environmental $B$. ambifaria AMMD and a few B. cepacia strains also produce HMAQs (Kilani-Feki et al., 2011, 2012; Mahenthiralingam et al., 2011). However, these latter studies required large concentrations of culture extracts, maybe explaining the absence of detection in the original studies. This suggests disparities in production levels between strains, possibly reflecting the lack of knowledge on the culture conditions directing the production of these secondary metabolites. Recently, Li et al. (2018) isolated new HMAQs from the culture broth of the environmental strain Burkholderia sp. MBAF1239. It is expected that more HMAQs congeners will be reported, with advances in metabolomic studies [e.g., see Okada et al. (2016)].

\section{REGULATION OF HMQ SYSTEM AND FUNCTION OF HMAQS}

Recently, a LysR-type transcriptional regulator named ScmR was found to positively influence the transcription of the hmqABCDEFG operon in B. thailandensis E264 (Mao et al., 2017). We found that ScmR has a potential 91.6\% homologous gene on a sequence portion and ortholog in B. ambifaria AMMD strain (BAMB_RS03575). While HAQs such as HHQ and $\mathrm{PQS}$ are QS autoinducers in $P$. aeruginosa, no hmqABCDEFG operon induction by HMAQs has been seen in B. ambifaria HSJ1 and B. thailandensis E264 (Pesci et al., 1999; Vial et al., 2008; Chapalain et al., 2017). However, the $h m q A B C D E F G$ operon appears indirectly repressed by the cep system, while production of acyl-HSL is up in a HMAQ-null mutant (Chapalain et al., 2017).

When present on the genome of Bcc strains, the hmqABCDEFG operon is located on the third chromosome except in B. ubonensis in which it is similar to the "pseudomallei" group, where it is located on chromosome 2. Its location on the third chromosome, defined by Agnoli et al. (2011) as a virulence megaplasmid, suggests that the hmqABCDEFG operon is involved in virulence in some Bcc species. For now, the only identified function of HMAQs is as antimicrobials (Kilani-Feki et al., 2011; Mahenthiralingam et al., 2011). The non-methylated HQNO plays role in B. thailandensis E264 by inhibiting cytochrome $b c 1$ and pyrimidine biosynthesis like in $P$. aeruginosa (Wu and Seyedsayamdost, 2017). Also, HMAQ-C9:1, the main congener produced by B. thailandensis, seems to dissipate the proton motive force and also inhibits pyrimidine biosynthesis act synergistically with HQNO to inhibit bacterial growth (Wu and Seyedsayamdost, 2017). The question remains open for the Bcc; in our previous work, HQNO family congeners were absent from B. ambifaria cultures, and we proposed this was explained by the absence of a PqsL homolog, the enzyme required for the biosynthesis of this family (Lépine et al., 2004; Vial et al., 2008). All our analyses performed since then on HMAQs produced by a range of $\mathrm{Bcc}$ strains support this assertion: the Bcc does not produce HQNO family H(M)AQs (unpublished). Since HMAQs affect $\mathrm{C}_{8}$-HSL production, this impacts QS phenotypes such as the production of siderophores and proteolytic activity (Vial et al., 2008). Because of the analogy with the HAQs, HMAQs might act as signaling molecules, be required to control many virulence factors and even have an immuno-modulating activity as described for other HAQs (Heeb et al., 2011; Price et al., 2018). In fact, in P. aeruginosa, HHQ synchronizes the bacterial population by inducing the production of PQS (Déziel et al., 2004). Furthermore, PQS and HHQ act as immunomodulator interacting with the peripheral blood mononuclear cells and the dendritic cells (Hooi et al., 2004; Skindersoe et al., 2009; Kim et al., 2010a,b). Price et al. (2018) have shown that three B. pseudomallei strains isolated from CF patients overexpressed the $h m q A B C D E F G$ operon compared to $B$. pseudomallei K96243. Theses strains could use HMAQs as antimicrobials in niche protection or to modulate the host 
immune response during infection (Price et al., 2018). Apart from signaling properties, HMAQ might have pharmaceutical potential by acting as an antimicrobial and as a cytotoxic antibiotic against IGF-I dependant cells in cancer progression (Mori et al., 2007; Li et al., 2018).

\section{CONCLUSION AND FUTURE DIRECTIONS}

Taking all these elements into consideration, it is important to understand the distribution of the $h m q A B C D E F G$ operon and its evolution within the Bcc. The hmqABCDEFG operon's presence is distributed within some species in the Bcc but not all of the sequenced strains in a given species have the operon. More sequencing data will be required to reach broader conclusions on the $h m q A B C D E F G$ operon distribution trends. Moreover, because available data suggest that clinical strains produce more HMAQs than the environmental ones, it will be necessary to assess this characteristic in more Bcc species, and in more strains by species.

Variant strains of $B$. ambifaria HSJ1 produce much less HMAQ than the wild type (Vial et al., 2009) even if they have $99.8 \%$ nucleotide identity with $B$. ambifaria HSJ1 and $B$. ambifaria AMMD genomes, suggesting that a negative regulator could control the Hmq system. It will be interesting to know if the QS regulation of HMAQ production could be a conserved feature in Bcc, as previously reported in $B$. ambifaria HSJ1, by testing different species (Chapalain et al., 2017).

\section{REFERENCES}

Agarwal, A., Kahyaoglu, C., and Hansen, D. B. (2012). Characterization of $\mathrm{HmqF}$, a protein involved in the biosynthesis of unsaturated quinolones produced by Burkholderia thailandensis. Biochemistry 51, 1648-1657. doi: 10.1021/bi201625w

Agnoli, K., Schwager, S., Uehlinger, S., Vergunst, A., Viteri, D. F., Nguyen, D. T., et al. (2011). Exposing the third chromosome of Burkholderia cepacia complex strains as a virulence plasmid. Mol. Microbiol. 83, 362-378. doi: 10.1111/j.1365-2958.2011.07937.x

Aguilar, C., Bertani, I., and Venturi, V. (2003). Quorum-sensing system and stationary-phase sigma factor $(r p o S)$ of the onion pathogen Burkholderia cepacia genomovar I type strain, ATCC 25416.s. Appl. Environ. Microbiol. 69, 1739-1747. doi: 10.1128/AEM.69.3.1739-1747.2003

Bertelli, C., Laird, M. R., Williams, K. P., Lau, B. Y., Hoad, G., Winsor, G. L., et al. (2017). IslandViewer 4: expanded prediction of genomic islands for larger-scale datasets. Nucleic Acids Res. 45, W30-W35. doi: 10.1093/nar/gkx343

Buchfink, B., Xie, C., and Huson, D. H. (2015). Fast and sensitive protein alignment using DIAMOND. Nat. Methods 12, 59-60. doi: 10.1038/nmeth.3176

Butt, A., Halliday, N., Williams, P., Atkins, H. S., Bancroft, G. J., and Titball, R. W. (2016). Burkholderia pseudomallei kynB plays a role in AQ production, biofilm formation, bacterial swarming and persistence. Res. Microbiol. 167, 159-167. doi: 10.1016/j.resmic.2015.11.002

Chapalain, A., Groleau, M.-C., Le Guillouzer, S., Miomandre, A., Vial, L., Milot, $\mathrm{S}$., et al. (2017). Interplay between 4-hydroxy-3-methyl-2-alkylquinoline and $\mathrm{N}$-acyl-homoserine lactone signaling in a Burkholderia cepacia Complex Clinical Strain. Front. Microbiol. 8:1648. doi: 10.3389/fmicb.2017. 01021

Choudhary, K., Hudaiberdiev, S., Gelencsr, Z., GonalvesCoutinho, B., Venturi, V., and Pongor, S. (2013). The organization of the quorum sensing
Our understanding of the prevalence, regulation and functions of HMAQs is limited. Apart from the antimicrobial activity, no function has been discovered yet. However, HMAQs may have similar roles to HAQs produced by $P$. aeruginosa based on their analogy (Vial et al., 2008; Price et al., 2018).

Future studies of the regulation of the $h m q$ system and the role of HMAQs are necessary to know if they play a role in virulence. This knowledge should provide a better understanding of Burkholderia especially in pathogens B. pseudomallei and Bcc strains.

\section{AUTHOR CONTRIBUTIONS}

PC: conception and design of the article and acquisition, analysis and interpretation of data; M-CG: experiments on hypothetical biosynthesis pathway; PC, M-CG, and ED: revision; ED: resources and funding.

\section{FUNDING}

This work was supported by grant MOP-142466 from the Canadian Institutes of Health Research (CIHR). ED holds the Canada Research Chair in Sociomicrobiology.

\section{SUPPLEMENTARY MATERIAL}

The Supplementary Material for this article can be found online at: https://www.frontiersin.org/articles/10.3389/fcimb. 2019.00033/full\#supplementary-material

luxI/R family genes in Burkholderia. Int. J. Mol. Sci. 14, 13727-13747. doi: 10.3390/ijms140713727

Darling, A. E., Mau, B., and Perna, N. T. (2010). progressiveMauve: multiple genome alignment with gene gain, loss and rearrangement. PLoS ONE 5:e11147. doi: 10.1371/journal.pone.0011147

Déziel, E., Lépine, F., Milot, S., He, J., Mindrinos, M. N., Tompkins, R. G., et al. (2004). Analysis of Pseudomonas aeruginosa 4-hydroxy-2alkylquinolines (HAQs) reveals a role for 4-hydroxy-2-heptylquinoline in cell-to-cell communication. Proc. Natl. Acad. Sci.U.S.A. 101, 1339-1344. doi: 10.1073/pnas.0307694100

Diggle, S. P., Lumjiaktase, P., Dipilato, F., Winzer, K., Kunakorn, M., Barrett, D. A., et al. (2006). Functional genetic analysis reveals a 2-Alkyl-4-quinolone signaling system in the human pathogen Burkholderia pseudomallei and related bacteria. Chem. Biol. 13, 701-710. doi: 10.1016/j.chembiol.2006.05.006

Drees, S., and Fetzner, S. (2015). PqsE of Pseudomonas aeruginosa acts as pathwayspecific thioesterase in the biosynthesis of alkylquinolone signaling molecules. Chem. Biol. 22, 611-618. doi: 10.1016/j.chembiol.2015.04.012

Drees, S. L., Li, C., Prasetya, F., Saleem, M., Dreveny, I., Williams, P., et al. (2016). PqsBC, a condensing enzyme in the biosynthesis of the Pseudomonas aeruginosa quinolone signal: crystal structure, inhibition, and reaction mecanism. J. Biol. Chem. 291, 6610-6624. doi: 10.1074/jbc.M115.708453

Dulcey, C. E., Dekimpe, V., Fauvelle, D.-A., Milot, S., Groleau, M.-C., Doucet, N., et al. (2013). The end of an old hypothesis: the Pseudomonas signaling molecules 4-hydroxy-2-alkylquinolines derive from fatty acids, not 3-ketofatty acids. Chem. Biol. 20, 1481-1491. doi: 10.1016/j.chembiol.2013.09.021

Eberl, L., and Vandamme, P. (2016). Members of the genus Burkholderia: good and bad guys. F1000Research 5:1007. doi: 10.12688/f1000research.8221.1

El-Banna, N., and Winkelmann, G. (2002). Pyrrolnitrin from Burkholderia cepacia: antibiotic activity against fungi and novel activities against streptomycetes. J. Appl. Microbiol. 85, 69-78. doi: 10.1046/j.1365-2672.1998.00473.x 
Farrow, J., Sund, Z., Ellison, M., Wade, D., Coleman, J., and Pesci, E. (2008). PqsE functions independently of PqsR-Pseudomonas quinolone signal and enhances the rhl quorum-sensing system. J. Bacteriol. 190, 7043-7051. doi: 10.1128/JB.00753-08

Farrow, J. M., and Pesci, E. C. (2017). Distal and proximal promoters co-regulate pqsR expression in Pseudomonas aeruginosa. Mol. Microbiol. 104, 78-91. doi: $10.1111 / \mathrm{mmi} .13611$

Folch, B., Dziel, E., and Doucet, N. (2013). Systematic mutational analysis of the putative hydrolase PqsE: toward a deeper molecular understanding of virulence acquisition in Pseudomonas aeruginosa. PLoS ONE 8:e73727. doi: 10.1371/journal.pone.0073727

Fuqua, C., and Greenberg, E. (1998). Self perception in bacteria: quorum sensing with acylated homoserine lactones. Curr. Opin. Microbiol. 1, 183-189. doi: 10.1016/S1369-5274(98)80009-X

Gallagher, L., McKnight, S., Kuznetsova, M., Pesci, E., and Manoil, C. (2002). Functions required for extracellular quinolone signaling by Pseudomonas aeruginosa. J. Bacteriol. 184, 6472-6480. doi: 10.1128/JB.184.23.6472-6480.2002

Gilligan, P. (1991). Microbiology of airway disease in patients with cystic fibrosis. Clin. Microbiol. Rev. 4, 35-51. doi: 10.1128/CMR.4.1.35

Gold, R., Jin, E., Levison, H., Isles, A., and Fleming, P. (1983). Ceftazidime alone and in combination in patients with cystic fibrosis: lack of efficacy in treatment of severe respiratory infections caused by Pseudomonas cepacia. J. Antimicrob. Chemother. 12(Suppl. A), 331-336. doi: 10.1093/jac/12.suppl_A.331

Govan, J., and Deretic, V. (1996). Microbial pathogenesis in cystic fibrosis: mucoid Pseudomonas aeruginosa and Burkholderia cepacia. Microbiol. Rev. 60, 539-574.

Govan, J., Doherty, C., Nelson, J., Brown, P., Greening, A., Maddison, J., et al. (1993). Evidence for transmission of Pseudomonas cepacia by social contact in cystic fibrosis. Lancet 342, 15-19. doi: 10.1016/0140-6736(93)91881-L

Heeb, S., Fletcher, M., Chhabra, S., Diggle, S., Williams, P., and Cmara, M. (2011). Quinolones: from antibiotics to autoinducers. FEMS Microbiol. Rev. 35, 247-274. doi: 10.1111/j.1574-6976.2010.00247.x

Hooi, D., Bycroft, B., Chhabra, S., Williams, P., and Pritchard, D. (2004). Differential immune modulatory activity of Pseudomonas aeruginosa quorum-sensing signal molecules. Infect. Immun. 72, 6463-6470. doi: 10.1128/IAI.72.11.6463-6470.2004

Huber, B., Riedel, K., Hentzer, M., Heydorn, A., Gotschlich, A., Givskov, M., et al. (2001). The cep quorum-sensing system of Burkholderia cepacia H111 controls biofilm formation and swarming motility. Microbiology 147(Pt 9), 2517-2528. doi: 10.1099/00221287-147-9-2517

Johnson, M., Zaretskaya, I., Raytselis, Y., Merezhuk, Y., McGinnis, S., and Madden, T. L. (2008). NCBI BLAST: a better web interface. Nucleic Acids Res. 36, W5-W9. doi: 10.1093/nar/gkn201

Juhas, M., Eberl, L., and Tmmler, B. (2005). Quorum sensing: the power of cooperation in the world of Pseudomonas. Environ. Microbiol. 7, 459-471. doi: 10.1111/j.1462-2920.2005.00769.x

Juhas, M., van der Meer, J. R., Gaillard, M., Harding, R. M., Hood, D. W., and Crook, D. W. (2009). Genomic islands: tools of bacterial horizontal gene transfer and evolution. Fems Microbiol. Rev. 33, 376-393. doi: 10.1111/j.1574-6976.2008.00136.x

Kang, Y., Carlson, R., Tharpe, W., and Schell, M. A. (1998). Characterization of genes involved in biosynthesis of a novel antibiotic from Burkholderia cepacia $\mathrm{BC} 11$ and their role in biological control of Rhizoctonia solani. Appl. Environ. Microbiol. 64, 3939-3947.

Kilani-Feki, O., Culioli, G., and Ortalo, M. (2011). Environmental Burkholderia cepacia strain Cs5 acting by two analogous alkyl-quinolones and a didecylphthalate against a broad spectrum of phytopathogens fungi. Curr. Microbiol. 62, 1490-1495. doi: 10.1007/s00284-011-9892-6

Kilani-Feki, O., Zouari, I., Culioli, G., Ortalo-Magne, A., Zouari, N., Blache, Y., et al. (2012). Correlation between synthesis variation of 2-alkylquinolones and the antifungal activity of a Burkholderia cepacia strain collection. World J. Microbiol. Biotechnol. 28, 275-281. doi: 10.1007/s11274-011-0 817-0

Kim, K., Kim, S.-H., Lépine, F., Cho, Y.-H., and Lee, G. R. (2010a). Global gene expression analysis on the target genes of PQS and HHQ in J774A.1 monocyte/macrophage cells. Microb. Pathog. 49, 174-180. doi: 10.1016/j.micpath.2010.05.009
Kim, K., Kim, Y. U., Koh, B. H., Hwang, S. S., Kim, S. H., Lépine, F., et al. (2010b). HHQ and PQS, two Pseudomonas aeruginosa quorumsensing molecules, down-regulate the innate immune responses through the nuclear factor-kappaB pathway. Immunology 129, 578-588. doi: 10.1111/j.1365-2567.2009.03160.x

Lawrence, J. G., and Ochman, H. (2002). Reconciling the many faces of lateral gene transfer. Trends Microbiol. 10, 1-4. doi: 10.1016/S0966-842X(01)02282-X

Lépine, F., Milot, S., Déziel, E., He, J., and Rahme, L. (2004). Electrospray/mass spectrometric identification and analysis of 4-hydroxy-2-alkylquinolines (HAQs) produced by Pseudomonas aeruginosa. J. Am. Soc. Mass Spectr. 15, 862-869. doi: 10.1016/j.jasms.2004.02.012

Lewenza, S., Conway, B., Greenberg, E. P., and Sokol, P. A. (1999). Quorum sensing in Burkholderia cepacia: identification of the LuxRI homologs CepRI. J. Bacteriol. 181, 748-756.

Lewenza, S., and Sokol, P. A. (2001). Regulation of ornibactin biosynthesis and $\mathrm{N}$-acyl-L-homoserine lactone production by CepR in Burkholderia cepacia. J. Bacteriol. 183, 2212-2218. doi: 10.1128/JB.183.7.2212-2218.2001

Li, D., Oku, N., Hasada, A., Shimizu, M., and Igarashi, Y. (2018). Two new 2-alkylquinolones, inhibitory to the fish skin ulcer pathogen Tenacibaculum maritimum, produced by a rhizobacterium of the genus Burkholderia sp. Beilstein J. Org. Chem. 14, 1446-1451. doi: 10.3762/bjoc.14.122

LiPuma, J. (1998). Burkholderia cepacia: management issues and new insights. Clin. Chest Med. 19, 473-486. doi: 10.1016/S0272-5231(05)70094-0

Loveridge, E. J., Jones, C., Bull, M., Moody, S., Kahl, M., Khan, Z., et al. (2017). Reclassification of the specialized metabolite producer Pseudomonas mesoacidophila ATCC 31433 as a member of the Burkholderia cepacia complex. J. Bacteriol. 199, e00125-e00117. doi: 10.1128/JB.00125-17

Mahenthiralingam, E., Song, L., Sass, A., White, J., Wilmot, C., Marchbank, A., et al. (2011). Enacyloxins are products of an unusual hybrid modular polyketide synthase encoded by a cryptic Burkholderia ambifaria Genomic Island. Chem. Biol. 18, 665-677. doi: 10.1016/j.chembiol.2011.01.020

Mao, D., Bushin, L. B., Moon, K., Wu, Y., and Seyedsayamdost, M. R. (2017) Discovery of $s c m R$ as a global regulator of secondary metabolism and virulence in Burkholderia thailandensis E264. Proc. Natl. Acad. Sci. U.S.A. 114, E2920E2928. doi: 10.1073/pnas.1619529114

McKenney, D., Brown, K., and Allison, D. (1995). Influence of Pseudomonas aeruginosa exoproducts on virulence factor production in Burkholderia cepacia: evidence of interspecies communication. J. Bacteriol. 177, 6989-6992. doi: 10.1128/jb.177.23.6989-6992.1995

Mori, T., Yamashita, T., Furihata, K., Nagai, K., Suzuki, K., Hayakawa, Y., et al. (2007). Burkholone, a new cytotoxic antibiotic against IGF-I dependent cells from Burkholderia sp. J. Antibiot. 60, 713-716. doi: 10.1038/ja.2007.92

Okada, B. K., Wu, Y., Mao, D., Bushin, L. B., and Seyedsayamdost, M. R. (2016). Mapping the trimethoprim-induced secondary metabolome of Burkholderia thailandensis. ACS Chem. Biol. 11, 2124-2130. doi: 10.1021/acschembio.6b00447

Pesci, E. C., Milbank, J. B. J., Pearson, J. P., McKnight, S., Kende, A. S., Greenberg, E. P., et al. (1999). Quinolone signaling in the cell-to-cell communication system of Pseudomonas aeruginosa. Proc. Natl. Acad. Sci. U.S.A. 96, 11229-11234. doi: 10.1073/pnas.96.20.11229

Pettersen, E. F., Goddard, T. D., Huang, C. C., Couch, G. S., Greenblatt, D. M., Meng, E. C., et al. (2004). UCSF Chimera-a visualization system for exploratory research and analysis. J. Comput. Chem. 25, 1605-1612. doi: 10.1002/jcc.20084

Price, E. P., Viberg, L. T., Kidd, T. J., Bell, S. C., Currie, B. J., and Sarovich, D. S. (2018). Transcriptomic analysis of longitudinal Burkholderia pseudomallei infecting the cystic fibrosis lung. Microb. Genomics 43, 465-414. doi: 10.1099/mgen.0.000194

Rampioni, G., Falcone, M., Heeb, S., Frangipani, E., Fletcher, M. P., Dubern, J.-F., et al. (2016). Unravelling the genome-wide contributions of specific 2-alkyl4-quinolones and PqsE to quorum sensing in Pseudomonas aeruginosa. PLoS Pathog. 12:e1006029. doi: 10.1371/journal.ppat.1006029

Rampioni, G., Pustelny, C., Fletcher, M. P., Wright, V. J., Bruce, M., Rumbaugh, K. P., et al. (2010). Transcriptomic analysis reveals a global alkyl-quinolone-independent regulatory role for PqsE in facilitating the environmental adaptation of Pseudomonas aeruginosa to plant and animal hosts. Environ. Microbiol. 12, 1659-1673. doi: 10.1111/j.1462-2920.2010.0 2214.x 
Ravenhall, M., Škunca, N., Lassalle, F., and Dessimoz, C. (2015). Inferring horizontal gene transfer. PLoS Comput. Biol. 11:e1004095. doi: 10.1371/journal.pcbi.1004095

Richau, J. A., Leitão, J. H., Correia, M., Lito, L., Salgado, M. J., Barreto, C., et al. (2000). Molecular typing and exopolysaccharide biosynthesis of Burkholderia cepacia: isolates from a portuguese cystic fibrosis center. J. Clin. Microbiol. 38, 1651-1655.

Sawana, A., Adeolu, M., and Gupta, R. S. (2014). Molecular signatures and phylogenomic analysis of the genus Burkholderia: proposal for division of this genus into the emended genus Burkholderia containing pathogenic organisms and a new genus Paraburkholderia gen. nov. harboring environmental species. Front. Genet. 5:429. doi: 10.3389/fgene.2014.00429

Siguier, P., Perochon, J., Lestrade, L., Mahillon, J., and Chandler, M. (2006). ISfinder: the reference centre for bacterial insertion sequences. Nucleic Acids Res. 34, D32-D36. doi: 10.1093/nar/gkj014

Skindersoe, M. E., Zeuthen, L. H., Brix, S., Fink, L. N., Lazenby, J., Whittall, C., et al. (2009). Pseudomonas aeruginosa quorum-sensingsignal molecules interfere with dendritic cell-induced T-cell proliferation. FEMS Immunol. Med. Microbiol. 55, 335-345. doi: 10.1111/j.1574-695X.2008.00533.x

Speert, D. P., Bond, M., Woodman, R. C., and Curnutte, J. T. (1994). Infection with Pseudomonas cepacia in chronic granulomatous disease: role of nonoxidative killing by neutrophils in host defense. J. Infect. Dis. 170, 1524-1531. doi: 10.1093/infdis/170.6.1524

Stewart, P. S., and Costerton, J. W. (2001). Antibiotic resistance of bacteria in biofilms. Lancet 358, 135-138. doi: 10.1016/S0140-6736(01)05321-1

Surez-Moreno, Z. R., Caballero-Mellado, J., Coutinho, B. G., Mendona-Previato, L., James, E. K., and Venturi, V. (2012). Common features of environmental and potentially beneficial plant-associated Burkholderia. Microb. Ecol. 63, 249-266. doi: 10.1007/s00248-011-9929-1

Vial, L., Chapalain, A., Groleau, M.-C., and Déziel, E. (2011). The various lifestyles of the Burkholderia cepacia complex species: a tribute to adaptation. Environ. Microbiol. 13, 1-12. doi: 10.1111/j.1462-2920.2010.02343.x

Vial, L., Groleau, M.-C., Dekimpe, V., and Déziel, E. (2007). Burkholderia diversity and versatility: an inventory of the extracellular products. J. Microbiol. Biotechnol. 17, 1407-1429.

Vial, L., Groleau, M.-C., Lamarche, M. G., Filion, G., Castonguay-Vanier, J., Dekimpe, V., et al. (2009). Phase variation has a role in Burkholderia ambifaria niche adaptation. ISME J. 4, 49-60. doi: 10.1038/ismej.2009.95

Vial, L., Lépine, F., Milot, S., Groleau, M.-C., Dekimpe, V., Woods, D. E., et al. (2008). Burkholderia pseudomallei, B. thailandensis, and B. ambifaria produce 4-hydroxy-2-alkylquinoline analogues with a methyl group at the 3 position that is required for quorum-sensing regulation. J. Bacteriol. 190, 5339-5352. doi: 10.1128/JB.00400-08

Wade, D. S., Calfee, M. W., Rocha, E. R., Ling, E. A., Engstrom, E., Coleman, J. P., et al. (2005). Regulation of Pseudomonas quinolone signal synthesis in Pseudomonas aeruginosa. J. Bacteriol. 187, 4372-4380. doi: 10.1128/JB.187.13.4372-4380.2005

Winsor, G. L., Khaira, B., Van Rossum, T., Lo, R., Whiteside, M. D., and Brinkman, F. S. L. (2008). The Burkholderia Genome Database: facilitating flexible queries and comparative analyses. Bioinformatics 24, 2803-2804. doi: 10.1093/bioinformatics/btn524

Wu, Y., and Seyedsayamdost, M. R. (2017). Synergy and target promiscuity drive structural divergence in bacterial alkylquinolone biosynthesis. Cell Chem. Biol. 24, 1437-1444.e3. doi: 10.1016/j.chembiol.2017.08.024

Xiao, G., Déziel, E., He, J., Lepine, F., Lesic, B., Castonguay, M. H., et al. (2006). MvfR, a key Pseudomonas aeruginosa pathogenicity LTTRclass regulatory protein, has dual ligands. Mol. Microbiol. 62, 1689-1699. doi: 10.1111/j.1365-2958.2006.05462.x

Yabuuchi, E., Kosako, Y., Oyaizu, H., Yano, I., Hotta, H., Hashimoto, Y., et al. (1992). Proposal of Burkholderia gen. nov. and transfer of seven species of the genus Pseudomonas homology group II to the new genus, with the type species Burkholderia cepacia (Palleroni and Holmes 1981) comb. nov. Microbiol. Immunol. 36, 1251-1275. doi: 10.1111/j.1348-0421.1992.tb0 2129.x

Zhang, Y.-M., Frank, M. W., Zhu, K., Mayasundari, A., and Rock, C. O. (2008). PqsD is responsible for the synthesis of 2,4-dihydroxyquinoline, an extracellular metabolite produced by Pseudomonas aeruginosa. J. Biol. Chem. 283, 28788-28794. doi: 10.1074/jbc.M804555200

Conflict of Interest Statement: The authors declare that the research was conducted in the absence of any commercial or financial relationships that could be construed as a potential conflict of interest.

Copyright (c) 2019 Coulon, Groleau and Déziel. This is an open-access article distributed under the terms of the Creative Commons Attribution License (CC BY). The use, distribution or reproduction in other forums is permitted, provided the original author(s) and the copyright owner(s) are credited and that the original publication in this journal is cited, in accordance with accepted academic practice. No use, distribution or reproduction is permitted which does not comply with these terms. 